\title{
Color-weak compensation using local affine isometry based on discrimination threshold matching
}

Rika Mochizuki, Takanori Kojima, Reiner Lenz and Jinhui Chao

\author{
Linköping University Post Print
}

\section{Tweet}

N.B.: When citing this work, cite the original article.

Original Publication:

Rika Mochizuki, Takanori Kojima, Reiner Lenz and Jinhui Chao, Color-weak compensation using local affine isometry based on discrimination threshold matching, 2015, Optical Society of America. Journal A: Optics, Image Science, and Vision, (32), 11, 2093-2103.

http://dx.doi.org/10.1364/JOSAA.32.002093

Copyright: Optical Society of America

http://www.osa.org/

Postprint available at: Linköping University Electronic Press

http://urn.kb.se/resolve?urn=urn:nbn:se:liu:diva-124139 


\title{
Color weak compensation using local affine isometry based on discrimination threshold matching
}

\author{
Rika MochizUki ${ }^{1}$, TAKanori KoJima ${ }^{2}$, Reiner Lenz ${ }^{3}$, And Jinhui ChaO ${ }^{2, *}$ \\ ${ }^{1}$ NTT Solution Lab, Tokyo, Japan \\ ${ }^{2}$ Dept. of Information and System Engineering, Chuo University, Tokyo, Japan \\ ${ }^{3}$ Dept. Science and Technology, Linköping University, SE-60174 Norrköping, Sweden \\ *Corresponding author:
}

\begin{abstract}
We develop algorithms for color weak compensation and color weak simulation based on Riemannian geometry models of color spaces. The objective function introduced measures the match of color discrimination thresholds of average normal observers and a colorweak observer. The developed matching process makes use of local affine maps between color spaces of color normal and color weak observers. The method can be used to generate displays of images that provide color normal and color weak observers with similar color-difference experience. It can also be used to simulate the perception of a color weak observer for color normal observers. We also introduce a new database of measurements of color discrimination threshold data for color normal and color weak observers obtained at different lightness levels in CIELUV space. The compensation methods include compensations of chromaticity using local affine maps between chromaticity planes of color normal and color weak observers, and 1D compensation on lightness. We describe how to determine correspondences between the origins of local coordinates in color spaces of color normal and color weak observers using a neighborhood expansion method. After matching the origins of the two coordinate systems a local affine map is estimated by solving a nonlinear equation, or singular-value-decomposition(SVD). We apply the methods to natural images and evaluate their performance using the semantic differential (SD) method.
\end{abstract}

OCIS codes: (330.0330) Vision, color, and visual optics, (330.1715) Color, rendering and metamerism, (330.1720) Color vision, (330.4060) Vision modeling

http://dx.doi.org/10.1364/ao.XX.XXXXXX

\section{INTRODUCTION}

Presenting a color image to observers so that their perception of the image is as similar as possible is a difficult problem. Methods to achieve this goal are important in human computer interface and have received a lot of interest due to recent rapid developments of visual media and wearable display technology.

The properties of color perception can vary considerably between observers. Usually one distinguishes between observers with normal color vision (we call them color-normal observers in the following) and different type of color-blind observers. But in reality the color vision properties of observers are varying widely between observers, they change with age and depend on health and viewing conditions, to name only a few important factors. There is no generally accepted definition of color weakness but in the following we will refer to persons which are not color blind but with a distinctive color vision deficiency as color weak observers. But it is important to note that there is the wide variation among color-weak observers ranging from very strong or near color-blind vision to slight color-weak or near-normal vision. We also remark that color perception cannot be measured quantitatively and there is therefore no objective criterion to measure the difference between color-normal vision and colorweak vision. It is therefore impossible to design algorithms that objectively compensate the difference.

A fundamental descriptor used to characterize color vision properties are color discrimination thresholds obtained by color matching. It is thus natural to use it to construct procedures that try to compensate color vision deficiencies. This data characterizes color vision by describing which region of color space produces the same color perception as a given test color. These regions can be approximated by ellipsoids around the test color and therefore one can model the color perception of an observer with the help of Riemann geometry. In the following we will model the color perception of observers using Riemann manifolds and use them to construct a criterion for color-weak compensation. The goal is the preservation of subjective color differences between color-normal and color-weak observers as 
measured by their Riemann geometries. A map preserving colordifferences or Riemannian distances between color spaces is known as an isometry and the task to compensate color-weak vision becomes to construct a color difference preserving map or an isometry. Information about methods based on Riemann geometry to characterize color spaces can be found in [1-5]. Earlier investigations of this approach can be found in [6-8].

In this paper we develop an algorithm to build a local isometry at neighborhoods of sampling points between two color spaces when the Riemann metric tensors (describing the local geometry) are available in both spaces. This amounts to matching the color discrimination thresholds of color-normal and colorweak observers. There is another way to build an isometry (see $[7,9,10]$ for details) using Riemann normal coordinate system and geodesics in both color spaces. The local approach represented here is easier to implement since it only requires linear algebra manipulations at each neighborhood, while the methods using geodesics requires the solution of second order ordinary differential equations and it also needs a smooth interpolation of the Riemann metric tensor. Two drawbacks of the current method are, firstly, that the estimation of the local isometry from observed data could result in ill-conditioned linear equations and, secondly, that one needs to find correspondences between neighborhoods and to determine the origins of corresponding local coordinates before estimating the local isometries between them. Both problems are not trivial in dimensions higher than one. We will show below that the first estimation problem is under-determined and therefore no unique solution can be found using a local isometry based on Riemann metric tensor information alone. The second problem is also directly related with the unobservability of color perception mentioned above.

In the following we first show a one dimensional color-weak model and compensation algorithms based on Brettel's color blind model along confusion lines [11]). Then we generalize the theory and algorithms to higher dimensional color spaces and address the above problems as follows: We construct the color weak compensation using a function that maps between the color spaces of the color-weak and the color-normal observer in such a way that it preserves the color differences as represented by the discrimination ellipsoids. We introduce new algorithms to determine such a local ellipsoid preserving map. We show that the construction requires the solution of a nonlinear equation or a singular-value- decomposition of the metric matrices for a restricted form of the function. The uniqueness of the local matrices is obtained using common reference vectors such as the invariant hues (as described in [11]) and continuity between neighboring local maps. The constructed functions are local and defined on patches. These patches must be pasted together in order to construct a global mapping. This requires the establishment of a correspondence between the patches in the two spaces. We also introduce a new neighborhood expansion algorithm to determine the images of the origins under the global isometry.

All these methods are based on the characterization of the color perception properties in the form of color discrimination data. We also describe a new database of threshold data measured at different lightness levels $L=30 ; 40 ; 50 ; 60 ; 70$ (in the CIELUV system). This is used in a compensation method of the type $2 \mathrm{D}+1 \mathrm{D}$ consisting of a $2 \mathrm{D}$ compensation by local affine isometries followed by a 1D compensation in the direction of lightness.

Evaluation of compensation methods is difficult due to the problems mentioned above. Here we will evaluate the pro- posed color-weak compensation methods based on the new measurement database in experiments where the performance is evaluated by the Semantic Differential (SD) method (described in [12]). We will show that we can indeed reduce the perceptual differences between color normal and color weak observers as measured by the correlation coefficients and distances in semantic differential evaluations.

\section{GEOMETRY OF COLOR SPACES AND COLOR- DIFFERENCE-PRESERVING MAPS}

A Riemannian manifold or a Riemann space is a space that looks locally approximately like a flat euclidean space but which usually has a very different global geometry. In a neighborhood of a point $x$ in such a Riemann space $C$ we measure the length of the deviation $d x$ from $x$ as

$$
\|d x\|^{2}=d x^{T} G(x) d x
$$

where $G(x)$ is a possitive definite matrix smoothly varying with $x$. This matrix is known as the Riemann metric tensor and for $G=I$ (the identity matrix) we obtain a Euclidean space. The fact that a color space can be better modeled as a Riemann space rather than a Euclidean space was already noted by Helmholtz and Schrödinger [4] and was mentioned by Riemann himself when he introduced his new type of geometry. Color differences are distances in the color space and the color discrimination threshold at $x$ defines the unit sphere centered at $x$ in the geometry of the observer. The corresponding metric tensor $G(x)$ is determined by psychophysical color matching experiments. Examples of such regions that describe the color discrimination properties of an observer are the well-known MacAdam ellipsoids.

The distance between two color vectors $x_{1}$ and $x_{2}$ in the color space is defined as the length of the shortest curve (geodesic) connecting the two points $\gamma_{12}$, it is defined as:

$$
d\left(x_{1}, x_{2}\right)=\int_{\gamma_{12}}\|d x\|=\int_{\gamma_{12}} \sqrt{d x^{T} G(x) d x}
$$

For color spaces $C_{k}$ with the Riemann metric $G_{k}(x), k=1,2$, a map $f$ from $C_{1}$ to $C_{2}$ which maps $x \in C_{1}$ to $y=f(x) \in C_{2}$, is called a local isometry if it preserves local distances everywhere. A map preserving large color-differences is called global isometry, which means that the distance between any pair of points in one space is equal to the distance between the corresponding pair of points in the other space. A global isometry is also local isometry and vice versa.

Given the two color spaces $C_{1}, C_{2}$ of a color-normal and a color-weak observer, this means that if we can match the thresholds at every corresponding pair of color points in the two space, then the small color differences are adjusted to be always the same everywhere and the color difference between any corresponding pair of colors is also identical.

In order to achieve equivalent perceptions for different observers we should try to preserve these relative differences. If we want to generate identical color perceptions for a color-weak observer and a color-normal observer we should present to both color distributions with the same color differences between every pair of colors measured in their personal color spaces. This provides us with an objective criterion for color-weak compensation and color transformation for different individuals in general. In other words: the equivalence between color perceptions is achieved by generating an equivalence of color spaces as Riemann spaces. 
In the following we define the color difference preserving map which transforms space $C_{n}$ of color-normal observers to $C_{w}$ of a color-weak observer (the "color-weak" map) as follows:

$$
w: C_{n} \longrightarrow C_{w}, \quad x \longmapsto y=w(x)
$$

The color matching experiment of the color normal observer determines the discrimination thresholds which in turn define the metric tensor $G_{n}(x)$ of the color space of the color-normal observer. In the same way the matching experiment for the color weak observer results in the metric tensor $G_{w}(y)$ in the color weak space. The color weak mapping is given by $w$ with $y=$ $w(x)$ and we therefore find the threshold matching condition:

$$
G_{w}(y)=\left(D_{w}\right)^{T} G_{n}(x) D_{w}
$$

with the Jacobian matrix $D_{w}$ of $w$ such that $d y=D_{w} d x$. The compensation of the color-weakness is given by the inverse map of the color-weak map $w$. On the other hand, applying $w$ to the input image and showing it to color-normal observers will provide them with the same experience, i.e. the simulation of the color-weak vision.

\section{COLORWEAK COMPENSATION BY DISCRIMINATION THRESHOLD MATCHING}

Human color perception depends on the existence of the three $\mathrm{L}, \mathrm{M}, \mathrm{S}$ cones on the retina. It is known that a model of colorblind vision can be represented by a projection in LMS space along the $\mathrm{L}$ axis for protanopia and the $\mathrm{M}$ axis for deuteranopia. Since color-blind observers cannot discriminate between colors in the directions of the $\mathrm{L}$ axis for protanopia and the $\mathrm{M}$ axis for deutanopia, the straight lines parallel to the L or M axis are called the confusion lines. The color blind model by Brettel et al [11] determines the color stimuli perceived by color blind observers, which consists of the two planes in LMS space. The two planes are spanned by three invariant hues which are perceived equally by both, color-normal and color-blind observers.

We first consider a linear color-weak model and compensation based on Brettel's model (which will later be generalized). (Fig.1). We denote $Q, Q^{\prime}$ as perception of color-normals and color-blinds, $Q^{\prime}$ is projection onto the color-blind planes along the $\mathrm{L}$ axis for protanopia and the $\mathrm{M}$ axis for deutanopia, which are in correspondence to the confusion lines. For every $3 \mathrm{D}$ color vector $\boldsymbol{R}$, only the $1 \mathrm{D}$ length along the confusion line passing through $R$ is of our interest, thus we will use the scalar $R$ to represents $R$ as its position on the confusion line.

In our linear color-weak model, every stimulus $Q$ is mapped by a color-weak map $w$ towards the color blind plane however without reaching it. Assuming that the color weak map $w$ is a linear map, then $Q^{\prime \prime}$ as the perception of color-weak observer can be described as the image of $Q$ under $w$ :

$$
\begin{aligned}
& Q^{\prime \prime}=w(Q)=\omega Q^{\prime}+(1-\omega) Q \quad(0 \leq \omega<1) \\
& Q^{\prime \prime}=w(Q)=Q^{\prime}+(1-\omega)\left(Q-Q^{\prime}\right)
\end{aligned}
$$

Here $\omega$ is called the color-weak index which indicates the degree of color-weakness. The observer is completely color-blind if $\omega=1$ and color-normal if $\omega=0$. As shown in Fig. $1, Q^{\prime}$ is moved the reduced distance $\left(Q^{\prime}-Q\right)$ by a factor of $1-\omega$.

The color map $w$ simulates color-weak vision when applied to the original image, the compensation is obtained from the inverse map of $w$ :

$$
P=w^{-1}(Q)=Q^{\prime}+\frac{1}{(1-\omega)}\left(Q-Q^{\prime}\right)
$$

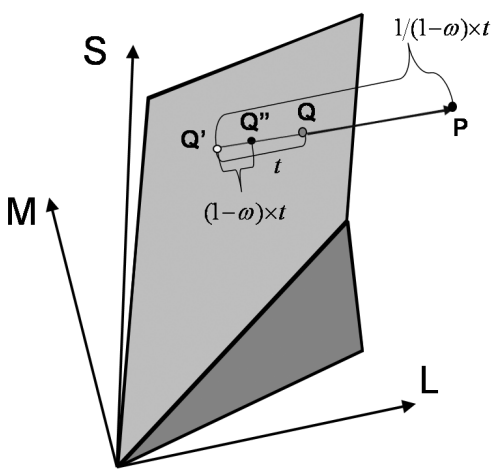

Fig. 1. Linear color-weak map along confusion lines

The color-weak index $\omega$ cannot be measured directly but it can be computed from the threshold matching data as follows: First, the Jacobian of color-weak map $w$ is given by

$$
D_{w}=1-\omega .
$$

and if $\alpha_{n}, \alpha_{\omega}$ are the thresholds of the color-normal and the color-weak along the confusion line then we find from Eq, (4):

$$
\begin{aligned}
\alpha_{n} & =(1-\omega) \alpha_{w} \\
\omega & =1-\frac{\alpha_{n}}{\alpha_{w}}
\end{aligned}
$$

This global compensation map is not sufficient in general as can be seen from Figure 2. Here we show the thresholds of normal and color-weak observers along confusion lines (the measurement procedure will be described in detail later). We see the color-weakness has large variation not only among individuals but among different color stimuli for the same observer as well. Since the assumption of linearity of the color-weak map is equivalent to that the color-weak index $\omega$ is a constant on a confusion line, the color-weak map is therefore in general not a linear map. We will now derive a generalized version in which the compensation mapping varies locally.
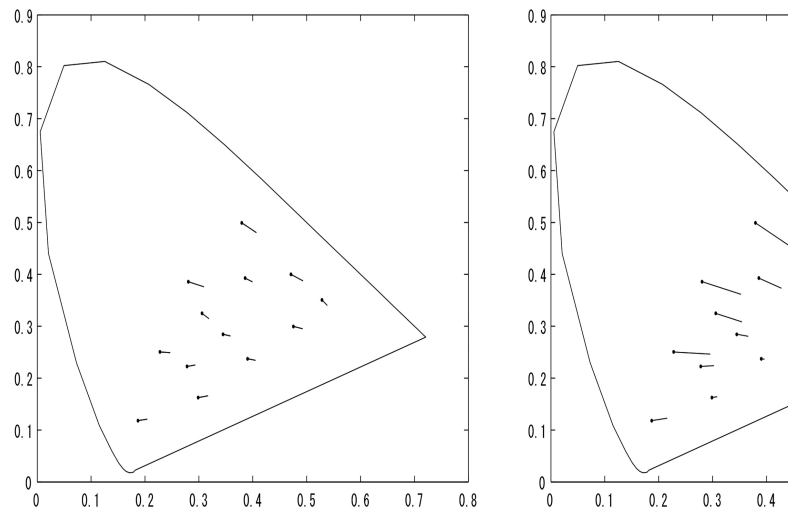

Fig. 2. Thresholds of normal and color-weak observers along confusion lines

From the threshold matching criterion, the Jacobian of the color-weak map needs to satisfy the following condition (apply- 
ing Eq.(4)), where $\omega(Q)$ is the color-weak index at $Q$ :

$$
D_{w}(Q)=\frac{\alpha_{n}\left(Q^{\prime \prime}\right)}{\alpha_{w}(Q)}=: 1-\omega(Q)
$$

The generalized color-weak and compensation maps (generalizing Eqs. 5 and 6) are obtained as the integrals of the Jacobians

$$
\begin{aligned}
Q^{\prime \prime} & =w(Q)=Q^{\prime}+\int_{Q^{\prime}}^{Q}(1-\omega(x)) d x \\
P & =w^{-1}(Q)=Q^{\prime}+\int_{Q^{\prime}}^{Q} \frac{1}{1-\omega(y)} d y .
\end{aligned}
$$

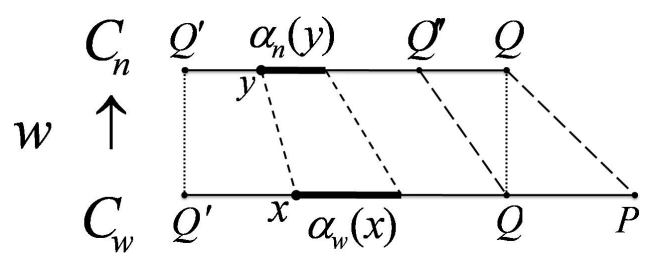

Fig. 3. Color-weak maps along confusion lines

To implement the color-weak map and the compensation map from the observed discrimination threshold data, choose the sampling points $\left\{x_{0}, x_{1}, x_{2}, \ldots\right\}$ on the confusion line in $C_{w}$ :

$$
x_{0}=Q^{\prime}, \quad x_{i+1}=x_{i}+\alpha_{w}^{(i)}
$$

Here, $\alpha_{w}^{(i)}:=\alpha_{w}\left(x_{i}\right), i=1,2, \ldots$ are the discrimination thresholds at sampled points $\left\{x_{i}\right\}$.

Similarly, the sampling points $\left\{y_{0}, y_{1}, y_{2}, \ldots\right\}$ on the confusion line of $C_{n}$ are defined using the discrimination threshold data $\alpha_{n}^{(j)}:=\alpha_{n}\left(y_{j}\right), j=1,2, \ldots:$

$$
y_{0}=Q^{\prime}, \quad y_{j+1}=y_{j}+\alpha_{n}^{(j)}
$$

Then under the color-weak map, one has the following correspondence between $x_{i}$ and $y_{j}$.

$$
\begin{aligned}
w\left(x_{k}\right) & =Q^{\prime}+\int_{Q^{\prime}}^{x_{k}}(1-\omega(x)) d x \\
& =x_{0}+\sum_{i=0}^{k-1} \int_{x_{i}}^{x_{i+1}}(1-\omega(x)) d x \\
& =y_{0}+\sum_{i=0}^{k-1} \alpha_{n}^{(i)}=y_{k} \quad k=0,1,2, \ldots
\end{aligned}
$$

The color-weak index at the $k$-th interval $\omega_{k}$ is defined by using the discrimination threshold of color-normals $\alpha_{n}^{(k)}$ and of the color-weak observer $\alpha_{w}^{(k)}$.

$$
\omega_{k}:=1-\frac{\alpha_{n}^{(k)}}{\alpha_{w}^{(k)}}
$$

Here $x_{I} \leq Q<x_{I+1}$ in $C_{w}$ and $y_{J} \leq Q<y_{J+1}$ in $C_{n}$.

Assuming $\alpha_{w}(x), x \in\left[x_{k-1}, x_{k}\right]$ in the $k$-th interval of $C_{w}$ is a constant equal to that on the right end of the interval $\alpha_{w}^{(k)}$ and the discrimination threshold in $k$-th interval in $C_{n}$ is a constant

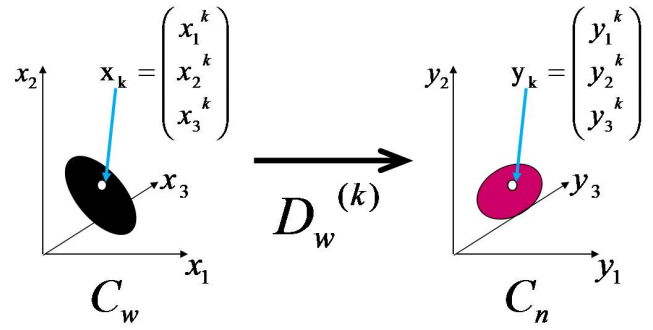

Fig. 4. Local color weak and compensation map

equal to $\alpha_{n}^{(k)}$, then the color-weak map and the compensation map can be realized by a sum of the discrimination thresholds on the confusion line:

$$
\begin{aligned}
& Q^{\prime \prime}=\sum_{i=0}^{I}\left(1-\omega_{i}\right)\left(x_{i+1}-x_{i}\right)=\sum_{i=0}^{I} \alpha_{n}^{(i)} \\
& P=\sum_{j=0}^{J} \frac{1}{1-\omega_{j}}\left(y_{j+1}-y_{j}\right)=\sum_{j=0}^{J} \alpha_{w}^{(j)}
\end{aligned}
$$

In fact, experimental data shows that the discrimination threshold ellipsoids do not simply expand along the confusion lines. It is reported in [13] that severe color-weak observers have discrimination threshold ellipsoids that expand along the confusion lines. On the other hand, for the color-weak observers with mild severity, the size and orientation of their discrimination threshold ellipsoids vary considerably. The main population of color-weak observers belongs to the latter group which implies the necessity of simultaneous compensation in more than one direction or a spatial compensation.

In particular, the colorweakness has to be described in higher dimensions which is much more complicated and the colorweak map can not be obtained in a closed form as in the 1D case. However, based on Riemann geometry, we can still build a global map which is locally defined at every point in the color spaces, based on the discrimination thresholds at these points.

Below, we show a high dimensional extension of the above theory and algorithms (Figure 4).

Assume we have a set of sampling points in 2D $C_{w}:\left\{x_{i}=\right.$ $\left.\left(x_{1}^{i}, x_{2}^{i}\right)^{T}\right\}$ or in 3D $C_{w}:\left\{x_{i}=\left(x_{1}^{i}, x_{2}^{i}, x_{3}^{i}\right)^{T}\right\}, i=1,2, \ldots, N$, corresponding to the set of the images of the sampling points into $C_{n}: y=w(x) \in C_{n},\left\{y_{i}=\left(y_{1}^{i}, y_{2}^{i}\right)^{T}\right\}$ in a $2 \mathrm{D} C_{n}$ and $\left\{y_{i}=\left(y_{1}^{i}, y_{2}^{i}, y_{3}^{i}\right)^{T}\right\}, i=1, \ldots, N$ in a $3 \mathrm{D} C_{n}$.

The colorweak map $w: C_{w} \longmapsto C_{n}$ can be linearly approximated in the neighborhoods of each sampling point onto the neighborhoods of their images, by the Jacobian matrix $D_{w}^{(k)}=D_{w}\left(x_{k}\right)$. which defines the local affine map between the neighborhood of $x_{k}$ and the neighborhood of its image $y_{k}=w\left(x_{k}\right)$

$$
y-y_{k}=D_{w}^{(k)}\left(x-x_{k}\right)
$$

The Jacobian matrix $D_{w}^{(k)}$ of $w$ is determined again by the local isometry or threshold matching condition (4):

$$
G_{n}^{(k)}=\left(D_{w}^{(k)}\right)^{T} G_{w}^{(k)} D_{w}^{(k)}
$$




\section{ESTIMATION OF THE LOCAL AFFINE ISOMETRY}

We assume first that pairs of color stimuli $x, y$ in two color spaces $C_{1}$ and $C_{2}$ are given and that these points are corresponding to each other under a (global) isometry. The local geometry is given by the metric tensors at the two points $G_{1}(x)$ and $G_{2}(y)$. We describe how to determine a local linear isometry which maps $x \in C_{1}$ to $y \in C_{2}$ such that the local geometry (i.e. the local color difference) at the neighborhood of $x \in C_{1}$ and $y \in C_{2}$ is preserved.

The local linear isometry is given by the Jacobian of the global isometry at $x$ which is a matrix $D_{f}$ which preserves the Riemann metric $G_{1}(x), G_{2}(y)$ as follows:

$$
G_{2}(y)=D_{f}^{T} G_{1}(x) D_{f}
$$

Such a local linear isometry is not unique. In the 2D case, this equation links $2 \times 2$ symmetric matrices resulting in threee independent scalar equations. The matrix of the local isometry has however four entries and we get multiple solutions. In $3 \mathrm{D}$ one has six equations and local isometries defined by nine entries.

Therefore we consider in the following a restricted form of $f$ which is consists of a scaling of the long and short axes of the ellipsoids and a rotation:

$$
\begin{aligned}
D_{f}=R \Lambda & =\left(\begin{array}{cc}
\cos \theta & \sin \theta \\
-\sin \theta & \cos \theta
\end{array}\right)\left(\begin{array}{ll}
a & 0 \\
0 & b
\end{array}\right) \\
& =\left(\begin{array}{cc}
a \cos \theta & b \sin \theta \\
-a \sin \theta & b \cos \theta
\end{array}\right)
\end{aligned}
$$

Introducing the new variables $X=a \cos \theta, Y=b \sin \theta$, $Z=-a \sin \theta, W=b \cos \theta$ we obtain a nonlinear equations in $X, Y, Z, W$ :

$$
X Y+Z W=0
$$

Furthermore we choose local coordinates as the eigen vectors of $G_{1}(x)$ and one can therefore assume that $G_{1}(x)$ is a diagonal matrix

$$
G_{1}(x):=\left(\begin{array}{cc}
\lambda_{1} & 0 \\
0 & \lambda_{2}
\end{array}\right), G_{2}(y):=\left(\begin{array}{cc}
g_{11}^{(2)} & g_{12}^{(2)} \\
g_{21}^{(2)} & g_{22}^{(2)}
\end{array}\right) .
$$

This leads to the following system of equations from Eq. (17) and Eq. (19) which is solved to obtain the entries of $D_{f}$ :

$$
\begin{aligned}
\lambda_{1} & =g_{11}^{(2)} X^{2}+2 g_{12}^{(2)} X Z+g_{22}^{(2)} Z^{2} \\
0 & =g_{11}^{(2)} X Y+2 g_{12}^{(2)}(X W+Y Z)+g_{22}^{(2)} Z W \\
\lambda_{2} & =g_{11}^{(2)} Y^{2}+2 g_{12}^{(2)} Y W+g_{22}^{(2)} W^{2} \\
0 & =X Y+Z W
\end{aligned}
$$

In the three-dimensional case the solutions can be obtained as follows: Use the eigenvectors and eigenvalues of the matrices $G_{k}$ and write $G_{k}=U_{k}^{T} D_{k} D_{k} U_{k}$ where the $U_{k}$ are rotation and the $D_{k}$ are diagonal matrices. We now have:

$$
\begin{aligned}
G_{2} & =U_{2}^{T} D_{2} D_{2} U_{2}=D_{f}^{T} G_{1} D_{f} \\
I & =D_{2}^{-1} U_{2} D_{f}^{T} U_{1}^{T} D_{1} D_{1} U_{1} D_{f} U_{2}^{T} D_{2}^{-1} \\
& =V^{T} V
\end{aligned}
$$

With $V=D_{1} U_{1} D_{f} U_{2}^{T} D_{2}^{-1}$ which must be a rotation matrix and any rotation matrix $V$ gives a solution. We get:

$$
D_{f}=U_{1}^{T} D_{1}^{-1} V U_{2} D_{2}
$$

Next we construct the global isometry by pasting together these local affine isometries that are defined in neighborhoods of every corresponding pairs of points at $C_{w}$ and $C_{n}$ and which can be described as local affine isometries or linear maps between the tangent spaces at $x_{i} \in C_{w}$ and $y_{i} \in C_{n}$ for $i=1, \ldots, N$. However, the computation of the affine part or the correspondence between $x$ and $y$ is not trivial.

Here we use a method we call neighborhood expansion to estimate these correspondences. We start with a known corresponding pair $O_{w} \in C_{w}$ and $O_{n} \in C_{n}$ with given metric tensors $G_{w}\left(O_{w}\right)$ and $G_{n}\left(O_{n}\right)$. A typical choice is a pair defined by the location of the white point D65. The two points are used as the origins in the above local coordinates. Then we first estimate the local linear isometry $D$ between two linear tangent spaces at $O_{w}$ and $O_{n}$ or the neighborhoods $N_{O_{w}}$ and $N_{O_{n}}$, as described above.

Next we choose the points $x_{i}, i=1, . ., I$ inside the neighborhood $N_{O_{w}}$ of $O_{w}$ which are going to be used as the origins of local coordinates of the second generation in $C_{w}$. These neighborhoods then expand from the neighborhood of $O_{w}$ as the first generation. Their images in $C_{n}$ under the local isometry $D$ can be found as

$$
y_{i}=O_{n}+D\left(x_{i}-O_{w}\right)
$$

which are used as the origins of the local coordinates in $C_{n}$ corresponding to the neighborhoods of $x_{i}$.

Now for the second generation origins one builds local isometries $D_{i}: C_{w} \supset N_{x_{i}} \longrightarrow N_{y_{i}} \subset C_{n}, i=1, . ., I$ based on the Riemann metric $G_{w}\left(x_{i}\right)$ and $G_{n}\left(y_{i}\right)$. This process is repeated to expand the neighborhoods and for every new generation of origins to build $D_{j i}: C_{w} \supset N_{x_{j i}} \longrightarrow N_{y_{j i}} \subset C_{n}, i=1, . ., N_{j}$ based on the Riemann metric $G_{w}\left(x_{j i}\right)$ and $G_{n}\left(y_{j i}\right)$. These local isometries will then eventually result in a global isometry from $C_{w}$ to $C_{n}$.

\section{MEASUREMENT OF COLOR DISCRIMINATION THRESHOLD DATA}

A basic tool in color vision research is the color matching experiment. At the beginning of such an experiment the observer is shown two colors side-by-side on the display. The observer is then asked to modify one of the colors (called the comparison color) until it matches the other color (the test color). A match concludes the color matching experiment. There are many variants of this basic scheme, mainly differing in the way the pair of test and comparison colors are chosen in these experiments (For a discussion of how different factors can lead to a variation of the observation parameters during a psychophysical experiment the interested reader might consult [14],[15]). Known factors that can lead to biased estimates are anticipation, adaptation or learning effects of the observers, fatigue and loss of interest. We try to avoid these as follows: (i) we used random ordering of the test color and comparison colors, (ii) the comparison color is chosen by first selecting one of fourteen random directions in the color space centered at the test color, (iii) finally a point on the selected line at a random distance from the test color is selected as the comparison color. In each direction, the discrimination is measured four times (at random points in time in the measurement sequence). The speed of the color changes of the comparison color is controlled by the movement of the mouse wheel or the number of key touches. This speed is also randomly changed. 
The illumination used is a Panasonic Hf premia fluorescent light and an HP DreamColor LP2480zx Professional Display is used as display. This monitor has a gamut which is slightly larger than the Adobe RGB gamut. The background is the neutral grey N5.5 from the Munsell color atlas. The distance between the monitor and the observer is $80 \mathrm{~cm}$. The display shows two frames of size $14 \times 14 \mathrm{~cm}$ each. The test color is shown on the left and the comparison color at the right. After four sessions the background neutral gray N5.5 is shown for seven seconds on the whole display.

All color computations are done in the CIELUV color space which is the color space of choice in color matching experiments using additive color displays such as monitors. The sampling points in CIELUV space are collected from five planes: $L=$ $30,40,50,60,70$. On each plane, a uniform grid of sampling points is selected. The number of selected points are; 9 points in $L=30,13$ points in $L=40,19$ points in $L=50,20$ points in $L=60$ and 16 points in $L=70$. This results in 77 points in the whole space.

\section{COLOR DISCRIMINATION THRESHOLD DATA}

The ellipsoids are then estimated from the observation data using the methods described in [8, 16] and [17]. The results for two observers, a D type color weak observer and a color-normal observer are shown in the following figures. The color weak observer was diagnosed as a $D$-type color-weak observer by the Anomaloscope.

The 77 threshold ellipsoids in CIELUV in the five levels of lightness have intersections with the $u^{*} v^{*}$ plane as ellipses. The threshold ellipsoids measured are shown in Fig. 5 for the colornormal and Fig. 6 for the color-weak observer. It can be observed that the threshold ellipsoids of the color-weak observers are larger in every lightness levels which suggest insensibility comparing with color-normal observers.

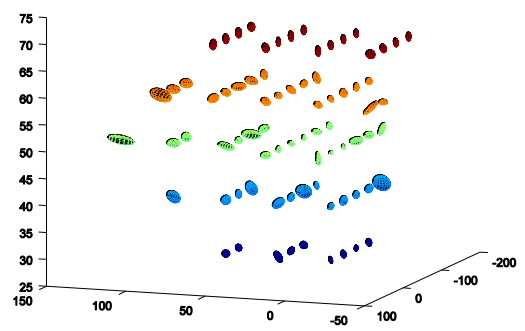

Fig. 5. Ellipsoids of color normal observer

The discrimination threshold ellipses as $2 \mathrm{D}$ intersection of ellipsoids with planes of constant L-values $L=30,40,50,60,70$ are shown here for $L=50,60$ in Fig. 7, 8 and Fig. 9, 10. One notices that the $D$-type color-weak observer has threshold ellipses expanded not only along the confusion lines of the $D$-type but also in other directions in every lightness level. The expansion varied also at each color which illustrates the complicated characteristics of color-weakness. These illustrates the necessity of high dimensional and color dependent compensation mentioned above.

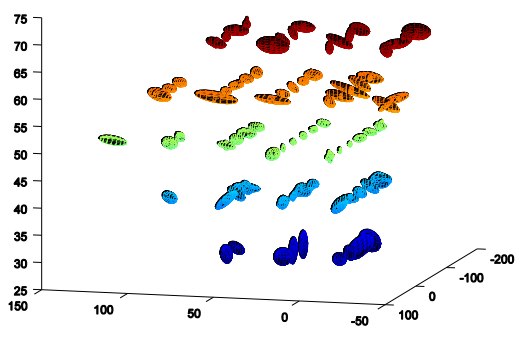

Fig. 6. Ellipsoids of color weak observer

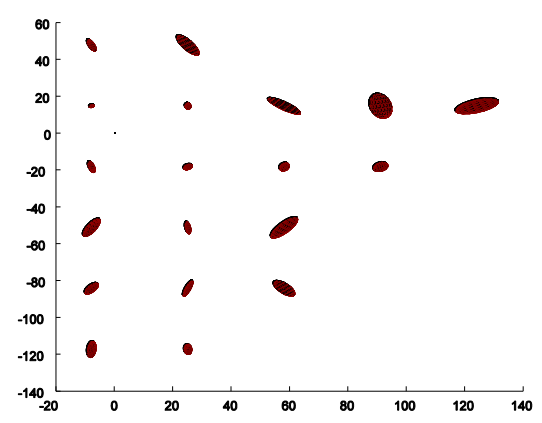

Fig. 7. Color normal discrimination threshold ellipses in $\mathrm{L}=50$

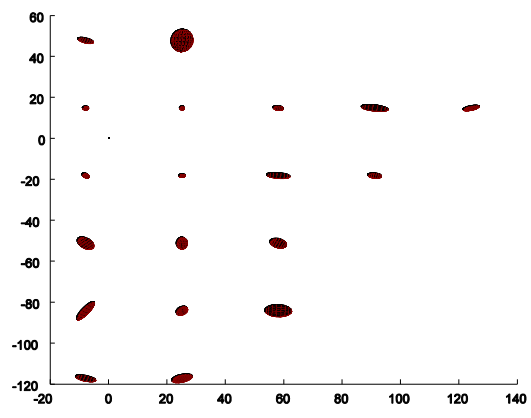

Fig. 8. Color weak discrimination threshold ellipses in $\mathrm{L}=50$

\section{EXPERIMENTS}

Figure 11 shows the simulation of color-weak vision, the original and the compensated image by the 1D color-weak map. Figure 12 shows the simulation of color-weak vision, the original and compensated image by the $2 \mathrm{D}$ color-weak map on the chromaticity plane. Here the color-weak map and compensation is described locally by $2 \times 2$ matrices and estimated from matching of threshold ellipses using Eq.(16).

In this case, the colorweak observer is not only a D-type as judged by Anomaloscope but also of a mild P-type as well. The $1 \mathrm{D}$ compensation along the confusion lines of D-type enhanced green alone as shown in Fig.11, which seemed also excessive. This is expected since the 1D model is not sufficient to fully describe his color-weakness. Meanwhile in the compensation based on the 2D model, both green and red hues are enhanced at the same time as shown in Fig.12.

In Fig. 13 to Fig. 15, we show three sets of simulation and 


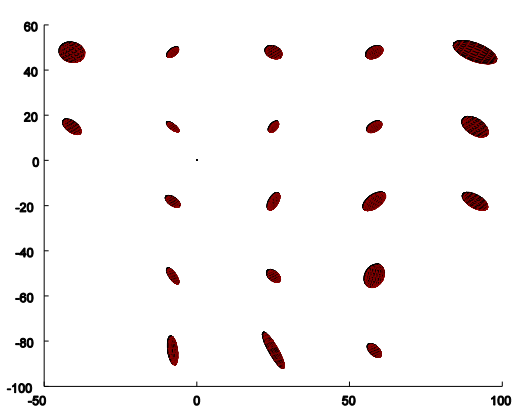

Fig. 9. Color normal discrimination threshold ellipses in $\mathrm{L}=60$

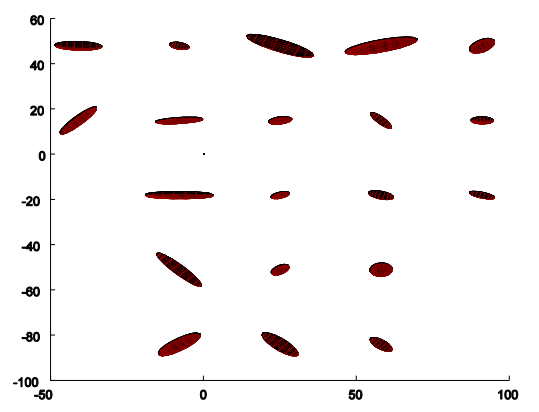

Fig. 10. Color weak discrimination threshold ellipses in $\mathrm{L}=60$

compensation results along with the original images. In these examples we consider the case of a color normal observer compared with a $D$-type or green insensitive color-weak observer. The original images where chosen to represent different general color properties: in Fig.13a the image consists mainly of rich variations of greens, the image in Fig. 14a contains mainly red and green tones and the image in Fig. 15a contains red green and blue regions.

The 2D color weak map or simulation map and the compensation map are applied to the original images on the five lightness levels. At the same time, 1D compensation and simulation are applied to the lightness axis. From the results one can see how the algorithm changes the color distributions to compensate or to simulate the deficencies of the color weak observer. In Fig. 15 we can see that the modifications result in band-like artifacts in the sky region of the image. Some possible causes of this effect will be discussed in a general context in Section 9. Here we only remark that the goal of these experiments is not primarily to produce good-looking results but to illustrate the properties of an implementation of the basic framework.

\section{SUBJECTIVE EVALUATION}

It is difficult to evaluate these methods directly. Here we illustrate our evaluation using the Semantic Differential (SD) method described in [12].

We selected 20 pairs of adjectives from the 76 pairs used in [12]. These pairs were chosen to represent more general visual properties of the images excluding those subjected to influence by adaptation and learning such as "natural/unnatural", "friendly/unfriendly". The selected pairs are listed in Table 1 and shown in the y-axes in Figures 16 to 21. Each pair represents opposite-meaning terms and a scale is set up with these two

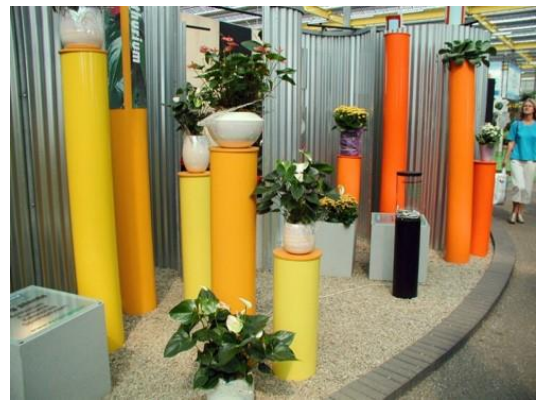

(a) Original image

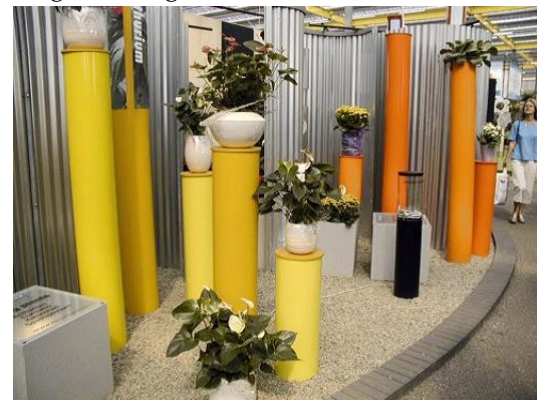

(b) $1 \mathrm{D}$ color-weak simulation

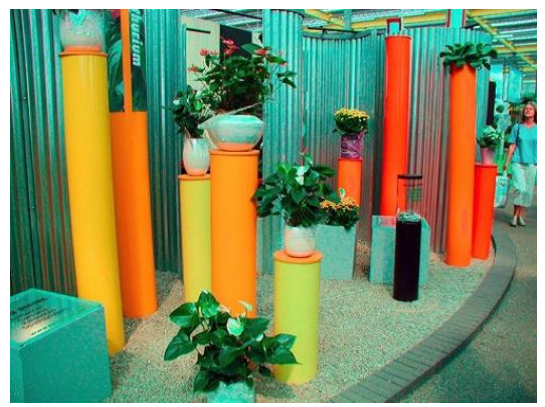

(c) $1 \mathrm{D}$ color-weak compensation

Fig. 11. Experiment with $1 D$ Compensation Method

adjectives at the opposite ends of such a scale. The observer is then asked to rate an image by putting a mark on one of the seven spaces along this scale. For example a score of one on the (hard-soft) scale would mean that the image is rated as very hard whereas a seven would mean it is rated as very soft. These scores are shown on the x-axes in Figures 16 to 21 . The scores for each of the pairs are illustrated in Figures 16 to 21 . As an example we can see that the color weak observers selected score 1 for adjective pair 10 in Figure 20 while color normal observers selected score 4 for the same pair. Comparing this with the same adjective pair in the compensated image Figure 21 we see that now the color weak and color normal observers more or less agree.

To evaluate the color-weak simulation, we compared the SD scores of the color-weak observer seeing the original images with the color-normal observer seeing the color-weak simulated images. The color-weak compensation is evaluated by comparison between the SD scores of the color-weak observer seeing the compensated image with the color-normal observer seeing the original images.

The normalized correlation coefficients and distances between the SD vectors for color-weak compensation as above are shown in Table 2, Table 3 and Table 4. All the correlation coefficients before compensation are negative which shown the 


\begin{tabular}{l|l|l|l|l|l|l|l|l|l|l} 
Wide & $\begin{array}{l}\text { Simple } \\
\text { Narrow }\end{array}$ & $\begin{array}{l}\text { Vague } \\
\text { Complicated }\end{array}$ & $\begin{array}{l}\text { Severe } \\
\text { Gentle }\end{array}$ & $\begin{array}{l}\text { Passive } \\
\text { Active }\end{array}$ & $\begin{array}{l}\text { Wet } \\
\text { Dry }\end{array}$ & $\begin{array}{l}\text { Strong } \\
\text { Weak }\end{array}$ & $\begin{array}{l}\text { Light } \\
\text { Heavy }\end{array}$ & $\begin{array}{l}\text { Young } \\
\text { Mature }\end{array}$ & $\begin{array}{l}\text { Opaque } \\
\text { Transparent }\end{array}$ \\
\hline Blunt & Masculine & Retrogressive & Excited & Close & Soft & Gorgeous & Cold & Intuitive & Rigid \\
Sharp & Feminine & Progressive & Calm & Far & Hard & Plain & Hot & Rational & Flexible
\end{tabular}

Table 1. Semantic Differential (SD) Adjectives

discrepancy between the color-normal and the color-weak vision, become positive after compensation. The distances between SD vectors are also reduced after compensation. This implies improvement of similarity between impressions of both observers for the same images, as a result of compensation on color distribution. Such effects are more apparent in the "Maple" with strong red green contrast than the green dominant "Forest". The results for the "Mountain" image is between these two cases.

Table 2. "Forest": SD scores

\begin{tabular}{|c|c|c|}
\hline & Correlation & distance \\
\hline Before compensation & -0.202395 & 0.321700 \\
\hline After compensation & 0.264135 & 0.278295 \\
\hline
\end{tabular}

Table 3. "Maple": SD score

\begin{tabular}{|c|c|c|}
\hline & Correlation & Distance \\
\hline Before compensation & -0.216530 & 0.500406 \\
\hline After compensation & 0.604984 & 0.286899 \\
\hline
\end{tabular}

Table 4. "Moutain": SD score

\begin{tabular}{|c|c|c|}
\hline & Correlation & Distance \\
\hline Before compensation & -0.721866 & 0.558297 \\
\hline After compensation & 0.238643 & 0.380957 \\
\hline
\end{tabular}

\section{CONCLUSIONS AND DISCUSSION}

We presented a novel color-weak compensation approach based on an objective criterion using the Riemann geometry of color spaces. We described methods using one-dimensional compensation along confusion lines and its high dimensional extension. We also introduced a new database characterizing the color vision properties of a color-normal and a color-weak observer.

These methods provide tools to simulate the perception of a given image by a color weak observer and to construct a color mapping that compensates the distortion of the color perception of a color weak observer in comparison to the perception of a color normal observer. In our experiments, especially in the subjective evaluation of the results, we saw that the proposed technique is useful in that context. We mentioned in Sec. 7 that the algorithm produced band-like artifacts in the compensation and simulation images. An extensive analysis of the origin of these effects is not possible here but we mention two fundamental problems with this approach that are certainly important in the understanding of this effect.

The first problem concerns the sampling of the color space and related to that the choice of interpolation methods. When collecting the threshold data we used a limited number of test colors from a regular pattern in CIELUV color space. Now all relevant color spaces in use today are constructed using the model of a color-normal standard observer. If we want to use the measurement data to deal with color deficient observers then we have to take into account both, the properties of the color normal population and the properties of the deficient color observers. Assume that our method requires to increase the resolution in some region of color space. This would lead to better interpolation properties. Of course this is not so easy in this case since each new sample point requires the rather complicated procedure of performing a number of color matching experiments.

The second problem is also related to the restrictions imposed by the fact that color technology is mainly designed with the color normal observer in mind. Color displays such as monitors produce the color pixels by additively mixing three basic light emitting elements. The colors produced, the gamut of the monitor, are therefore all located in the convex hull of the three primary channels. For test color near the boundary of the gamut the algorithm may require an out-of-gamut color that cannot be produced on this monitor, which may lead to artifacts. This problem is of course most prominent in the compensation case. Additionally one has to take into account the influence of the quantization error of standard digitial image formats like jpg and pdf. In the case of the printed version of this paper the restrictions of the printing process are also important.

The fact that the visible artifacts are in the blue-region is not too surprising since the geometry in the blue region of the color space is very different from the geometry in other regions, due to the number and distribution of the short-wavelength (blue) receptors in the retina. A detailed study of these effects lies outside the scope of this article but a better understanding is, of course, essential for improved future implementations.

Acknowledgments The authors wish to thank Prof. Miyoshi Ayama for valuable suggestions on experiments and Dr. Satoshi Oshima for assistance during in the research. This work was supported by Japan Society for the Promotion of Science (JSPS) KAKENHI Grant Number 23500156 and Institute of Science and Engineering, Chuo University. The support of the Swedish Research Council through a framework grant for the project Energy Minimization for Computational Cameras (2014-6227) and by the Swedish Foundation for Strategic Research through 


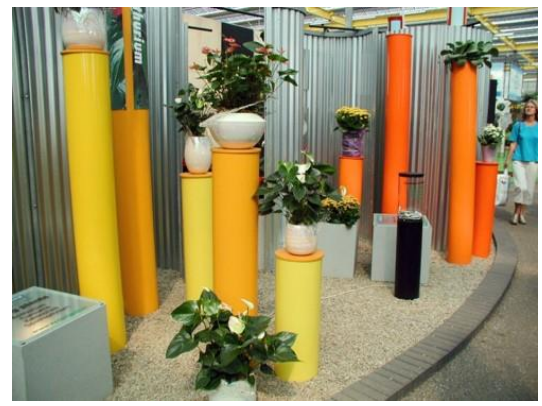

(a) Original image

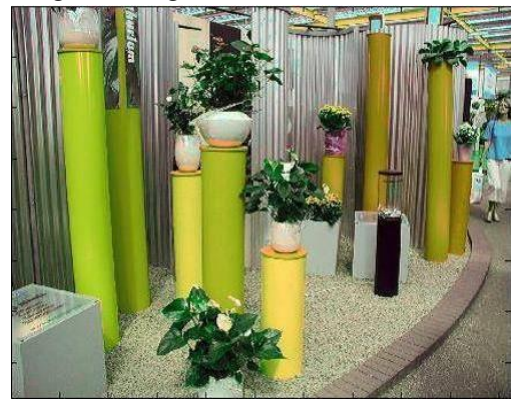

(b) 2D color-weak simulation

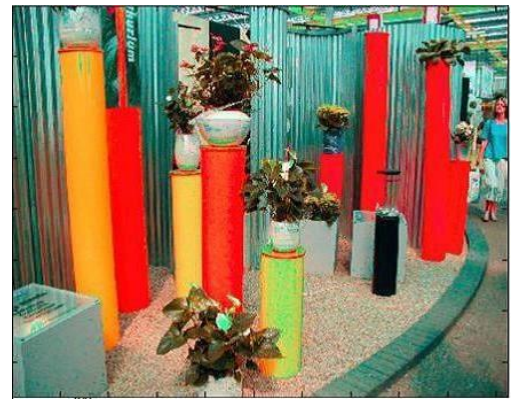

(c) 2D color-weak compensation

Fig. 12. Experiment with 2D Compensation Method

grant IIS11-0081 is gratefully acknowledged.

\section{REFERENCES}

1. W. D. Wright and F. Pitt, "Hue-discrimination in normal colour-vision," Proceedings of the Physical Society 46, 459 (1934).

2. W. Brown and D. MacAdam, "Visual sensitivities to combined chromaticity and luminance differences," JOSA 39, 808-823 (1949).

3. D. L. MacAdam, "Visual sensitivities to color differences in daylight," JOSA 32, 247-273 (1942).

4. G. Wyszecki and W. S. Stiles, Color science : concepts and methods, quantitative data and formulae (Wiley, New York, 1984), 2nd ed.

5. R. G. Kuehni, Color space and its divisions : color order from antiquity to the present (John Wiley \& Sons Inc., Hoboken, NJ, 2003).

6. J. Chao, I. Osugi, and M. Suzuki, "On definitions and construction of uniform color space," in "Conf. Colour in Graphics, Imaging, and Vision," (IS\&T, 2004), 1, pp. 55-60.

7. J. Chao, R. Lenz, D. Matsumoto, and T. Nakamura, "Riemann geometry for color characterization and mapping," in "Conf. Colour in Graphics, Imaging, and Vision," (IS\&T, 2008), 1, pp. 277-282.

8. R. Mochizuki, T. Nakamura, J. Chao, and R. Lenz, "Color-weak correction by discrimination threshold matching," in "Conf. Colour in Graphics, Imaging, and Vision," (IS\&T, 2008), 1, pp. 208-213.

9. S. Ohshima, R. Mochizuki, J. Chao, and R. Lenz, "Color reproduction using Riemann normal coordinates," in "Computational Color Imaging," (Springer, 2009), pp. 140-149.

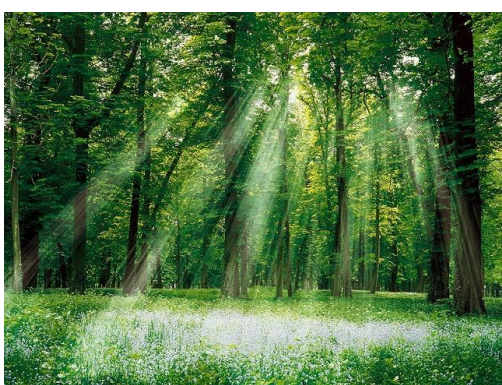

(a) Original

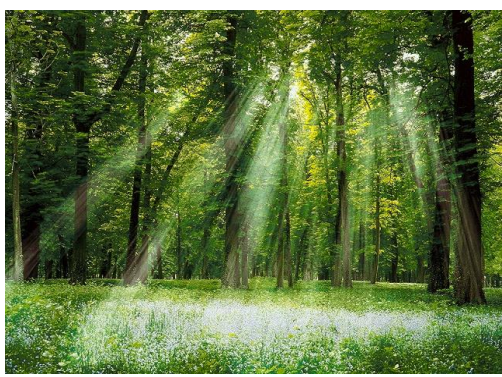

(b) Color-weak simulation

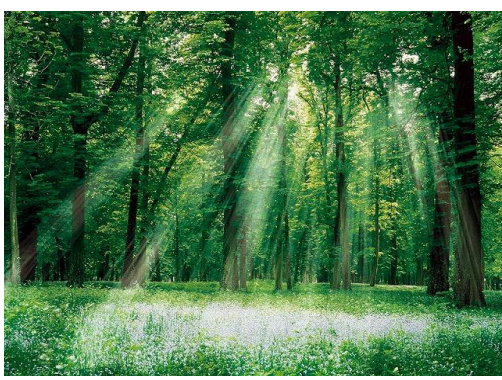

(c) Color-weak compensation

Fig. 13. "Forest" Example

10. S. Ohshima, R. Mochizuki, R. Lenz, and J. Chao, "Color-weakness compensation using Riemann normal coordinates." in "Proc. IEEE Int. Symposium on Multimedia," (2012), pp. 175-178.

11. H. Brettel, F. Vienot, and J. Mollon, "Computerized simulation of color appearance for dichromats," JOSAA 14, 2647-2655 (1997).

12. C. E. Osgood, G. J. Suci, and P. H. Tannenbaum, The measurement of meaning (University of Illinois Press, Urbana, 1957).

13. B. Regan, J. Reffin, and J. Mollon, "Luminance noise and the rapid determination of discrimination ellipses in colour deficiency," Vision research 34, 1279-1299 (1994).

14. R. Doll, P. Veltink, and J. Buitenweg, "Observation of time-dependent psychophysical functions and accounting for threshold drifts," Attention, Perception, and Psychophysics (2015). Article in Press.

15. I. Fründ, N. Haenel, and F. Wichmann, "Inference for psychometric functions in the presence of nonstationary behavior," Journal of Vision 11, 1-19 (2011).

16. R. Lenz, S. Oshima, R. Mochizuki, and J. Chao, "An invariant metric on the manifold of second order moments," in "IEEE 12th International Conference on Computer Vision Workshops," (IEEE, 2009), pp. 19231930.

17. R. Lenz, R. Mochizuki, and J. Chao, "Iwasawa decomposition and computational Riemannian geometry," in "20th International Conference on Pattern Recognition," (IEEE, 2010), pp. 4472-4475. 


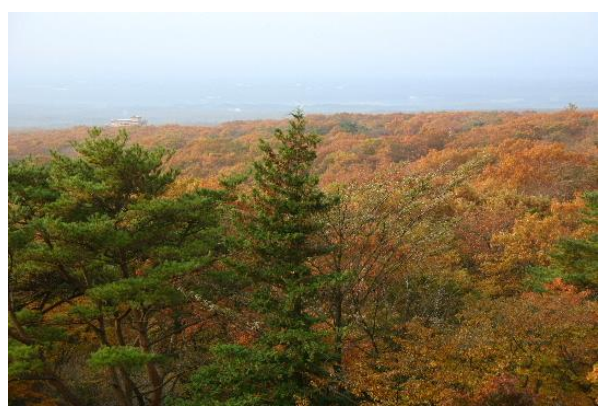

(a) Original

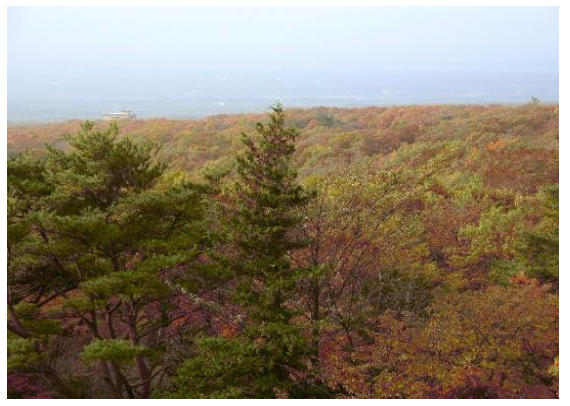

(b) Color-weak simulation

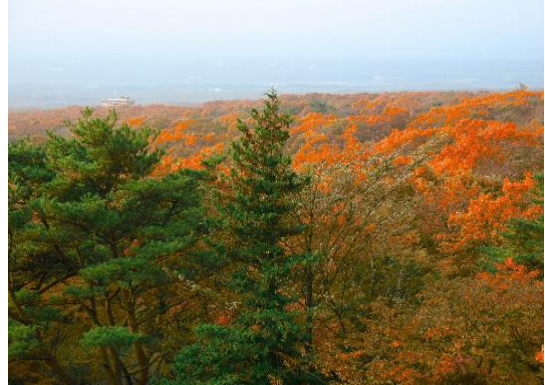

(c) Color-weak compensation

Fig. 14. "Maple" Example

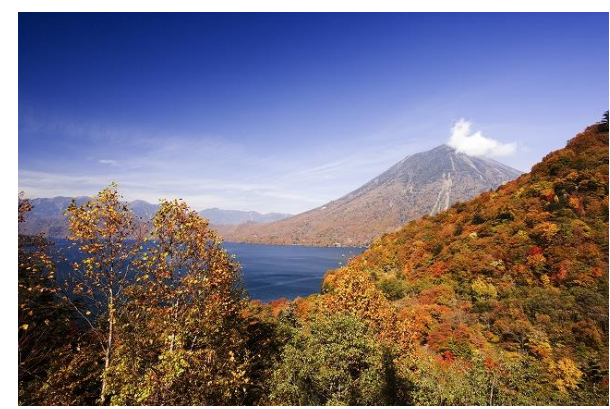

(a) Original

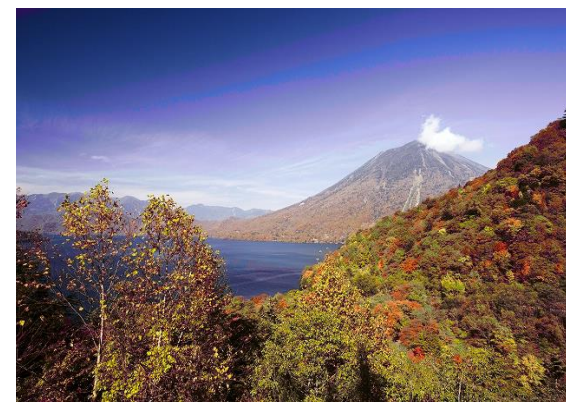

(b) Color-weak simulation

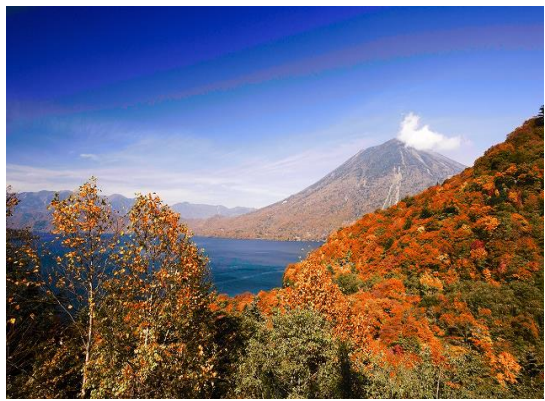

(c) Color-weak compensation

Fig. 15. "Mountain" Example

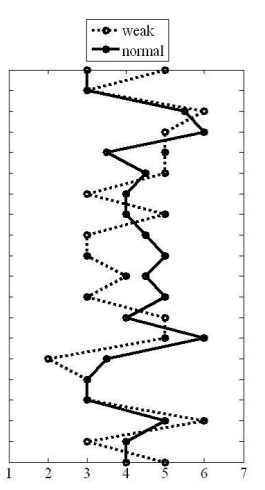

Fig. 16. Color-normal and color-weak views of original "Forest"

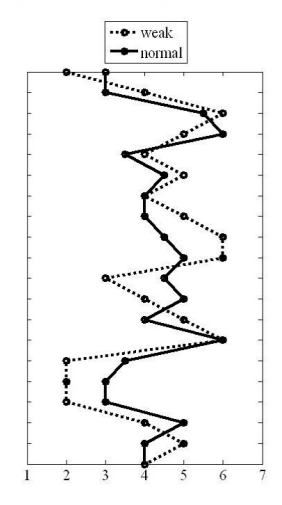

Fig. 17. Color-normal views original, color-weak views compensation of "Forest" 


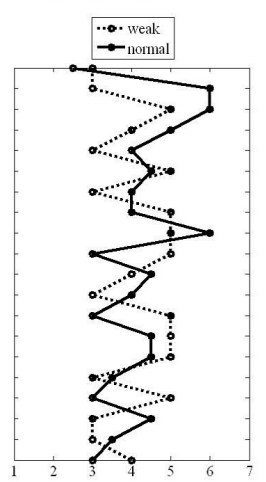

Fig. 18. Color-normal and color-weak views of original "Maple"

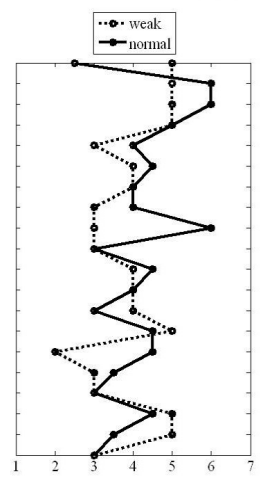

Fig. 19. Color-normal views original, color-weak views compensation of "Maple"

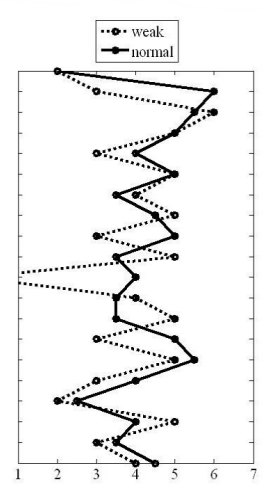

Fig. 20. Color-normal and color-weak views of original "Mountain"

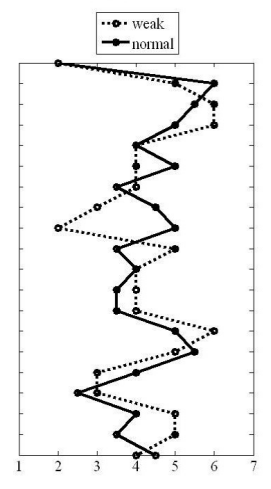

Fig. 21. Color-normal views original, color-weak views compensation of "Mountain" 